\title{
Improvement in Coverage Ratio using Overlap Sensing Shifted Node in Grid
}

\author{
Preeti \\ Department of Electrical Electronics and Communication Engineering \\ ITM University, Gurgaon, Haryana-122017
}

\begin{abstract}
In this paper we explores the competing issues of coverage effectiveness and power accessible in wireless sensor networks Nonstop capacity increase of distributed gridconnected system produces more obvious disturbance on the grid. The Monitoring network technology can run protection for the safety and stability of power grid operation, but sensor nodes of the monitoring network will fall to failure due to conservation interference. According to the performance degradation problem produced by nodes failure monitoring network, of natural selection based on random weight is proposed in this paper to optimize monitoring performance can restore the monitoring network by moving redundant nodes. Simulation results show the effectiveness of the proposed algorithm. Our lower energy shifting propagationloss model comprises with path loss function with random distributed shadowing, independent across with base stations. Our results are valid in the whole estate of ECOSR (Energy coverage overlap sensing ratio), in particular for ECOSR $<1$, where one discovers multiple coverage.
\end{abstract}

\section{Keywords}

Wireless sensor network; Coverage; Heterogeneous nodes; Stable election protocol.

\section{INTRODUCTION}

In the existence of the sensor network, coverage, localization, reliability and lifetime (when the final sensor dies) are the main design tests for which the work is still going on. Particularly in heterogeneous wireless sensor network, it is very challenging to estimation the coverage and lifetime of the network. There have been various existing standard protocols in heterogeneous network where the heterogeneity was taken either in sensing radius or in the energies of nodes. An idea to increase the coverage ratio as well as the lifetime of the senor network is optional in this paper by presenting flexibility in heterogeneous WSN, where the heterogeneity is measured in energy of nodes. We have selected the deployment in heterogeneous wireless sensor network with loco-mobility ability nodes which has different energies. The simulation results show that in our algorithm, maximum area is covered, and in addition, approximately nodes are in off state to preserve the energy. We reflect the nodes to cover large area while being consistent in sensing by saving energy. Our proposed algorithm's board is to schedule the sensor nodes in such a method that they can monitor an area efficiently. We associate our scheme with previously existing protocol named as stable election protocol, analyzing the network lifetime for different number of nodes in various areas and also the coverage throughout the lifetime.
A heterogeneous type of wireless sensor networks consists of huge number of standard nodes and a few heterogeneous nodes. The crucial function of normal nodes are to sense and supply data explosion, is reasonable and source-confined Maximum, terminated coverage may be suitable and preferred in nearly applications, such as consuming tiny sensor nodes to display a critical health situation in humans, and also in circumstances where unused of the sequences is conceivable when power is totally exhausted. Supreme, redundant coverage may also be necessary in applications using rough, environment sensor nodes for monitoring a planned soldierly scenario for a known duration of time. In these instances, the lifetime of the system is not exploited, and this is an completely satisfactory importance. At the other end of the variety, however, there are applications where maximum, redundant coverage is not the supreme reflection. A reduced coverage system may be entirely acceptable, as a tradeoff for massively extending the lifetime of the system. For example, any singularity that is not too different throughout an area; events, phenomenon that are slightly constant and one event at one point is not severely different than the same observed event at an together point. Put another way, in applications where the transaction of broadcasting to gain protracted system lifetime does not have severe negative consequences. Selected examples of these types of wonder are temperature, light, sound, atmospheric conditions.

\section{LITRATURE REVIEW}

Raymond Mulligan et.al [1] this paper can be utilized for the beginning or a summary of the work that has been done to that time, several terms and concepts are defined by the author and then it is shown that how they are being used in numerous research works. Coverage is one of the most influential and popular areas of research in the field of wireless sensor networks. Making certain that there is sufficient coverage in a sensor network is essential to obtain valid and substantial data. In this paper a broad review of the work that has been done in the coverage problems in wireless sensor networks is addressed thoroughly.

Chetan Chugh et.al [2] in this paper a brief photographic representation of wireless sensor nodes deployment in MATLAB software is given. WSNs have been broadly considered as one of the most crucial technologies in this era. This paper the path between the source and the destination nodes for reliable data delivery is accommodated. The hostile nodes are choosen on manual base. Using Dijstra algorithm, the substitute shortest route has been found. In addition, an RSA algorithm algorithm for public key cryptography has been implemented to safeguard the nodes from obstacle attacks. 
Wendi B.Heinzelman et.al [3] In this paper, authors develop and examine a low-energy adaptive clustering hierarchy $(\mathrm{LEACH})$. This is a protocol architecture for micro-sensor networks that merge the scheme of energy-efficient cluster based data routing and media access with each other and with the application-specific data aggregation to attain efficient performance in terms of latency, network lifetime, and application-perceived quality.

Wendi Rabiner Heinzelman et.al [4] in this paper, the work glance at communication protocols, which have outstanding impact on the whole energy decadence of the network. On the basis of the findings that the traditional protocols of minimum-transmission-energy, direct transmission, static clustering and multi-hop routing may not be advantageous for sensor networks. LEACH (Low-Energy Adaptive Clustering Hierarchy) is a clustering-based protocol that uses random rotation of local cluster base stations i.e. cluster-heads to equally distribute the energy load among the sensor nodes in the network was proposed.

M. J. Handy et.al [5] this paper highlights on decreasing the power utilization of wireless micro-sensor networks. Hence, a communication protocol called LEACH is transformed. LEACH's stochastic cluster head selection algorithm is extended by a deterministic component.

I. Saha Misra et.al [6] Clustering technique in sensor nodes is an productive technique to attain the objectives instead of the traditional routing protocols such as minimum transmission energy routing, direct transmission or other relevant protocols which are applicable for static networks. Reorganizing this approach, the work proposed an enhanced energy efficient adaptive clustering i.e.EEEAC protocol stand on the leftover energy of each node inside the network.

V.Loscri et.al [7] the authors proposed a improvement to a popular protocol for wireless sensor networks named as LEACH. This is created for the wireless sensor networks where the end user ought to remotely observe the scenario.

Yongcai Wang et.al [8] authors proposed EDAC (EnergyDriven Adaptive Clustering) protocol, which is a modification over LEACH in heterogeneous wireless sensor networks. Unlike the homogeneous networks, if LEACH is applied in heterogeneous networks, the average energy dissipation mechanism will out-turn in prior death of "powerless" nodes and cannot utilize the primacy of the "powerful" nodes.

Weilian Su et.al [9] Advancement in electronics and wireless communications has empower the development of small size and low cost wireless sensor networks. The sensor network has wide range in numerous application domain (e.g., health, medical, military, home). There are many and unalike technical issues that researchers are resolving for different applications at present time. The present state of sensor networks is presented in the paper, where results and answers are debated under respective protocol stack layers. In this article 9 open research topics are represented and aspire to spot recent notices and developments in the field of wireless sensor networks.

Paolo Baronti et.al [10] Wireless sensor networks are developing technology and hot area of research for low cost, remote monitoring of broad scenarios and targets, and their significance has been imposed by recent dispatch of the IEEE 802.15.4 standard for both physical and MAC layers and the imminent Zigbee standard for the network and the application layers. Because of the rapid advancement of research on networking data management, energy efficiency and security issues in wireless sensor networks, and the requirement to differentiate with the resolutions acquired in the standards promotes the requirement for a survey in this field.

\section{SYSTEM MODEL}

Enhancing Coverage Ratio Using Mobility in Heterogeneous Wireless Sensor Network[13] reflects on the idea of using mobile nodes to develop an algorithm to enhance coverage ratio of a network.

The structure model we accept that the sensor nodes are deployed randomly as per the coverage of sensor node in sensing field in the board area and all the sensor nodes have the same broadcast range $r$ with minimum coverage distance diagonally, left or right forwarding. The sensor estimate minimum and second higher energy would focus or call to transmit the node towards void are and may be minimum nodes in single grid. The information of neighboring nodes is received by each sensor node and decides on an instance local packet length in a distributed manner. We define the degree of average node $d$ to signify the connectivity of the whole network. For suitability an event area is a circle with radius 1.

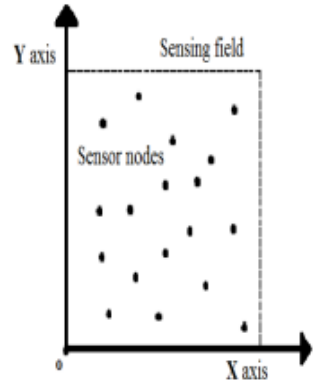

(a)

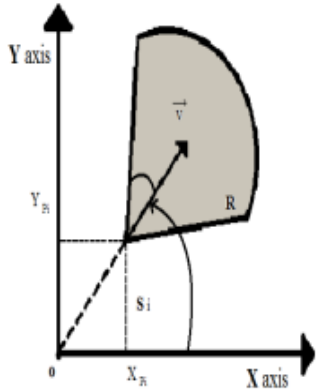

(b)
Fig 1: Sensing field and directional sensing field

The recommended adaptive system is predictable to achieve effectively even with dissimilar incident region shapes. So, each sensor node follows the advance node energy consumption, and cross validate frequent pattern of energy consumption of sensor node at the time of rounds. Although a week/dead sensor or an event may create packet loss for remaining near neighbor nodes, and hence they are in different established on the readings of a single sensor node and the remaining sensor calculations are expected to be binary, without loss of splitting network area.

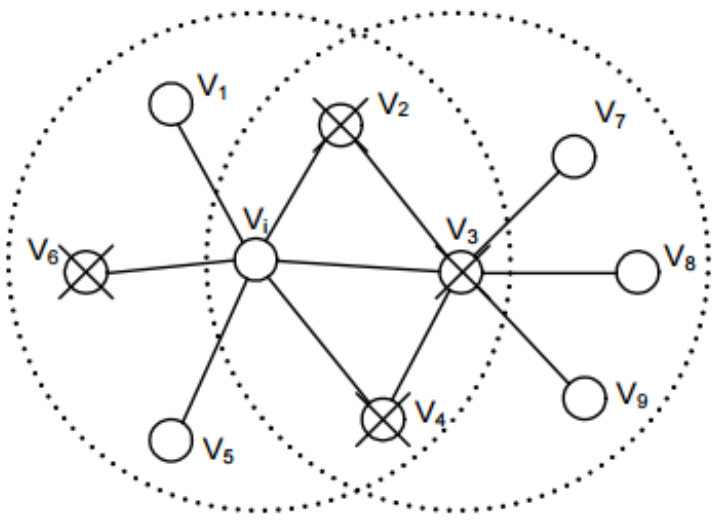

Fig 2: Node failing to pass the threshold level due to neighboring faulty nodes 


\section{PROPOSED IMPLEMENTATION}

1. The phases for lower energy node attention consumption formula and two types of essential to estimate the k-coverage probability in a network with log-normal shadowing (though the shadowing distribution can be slightly arbitrary and without coverage sensing area of higher energy shifting nodes.

2. We use a quadrature method or a simple analytic formula for coverage sense with advance node energy.

3. The more compound high-dimensional essential uses quadrature methods for low dimensions and quasi-random integration for higher $(n>2)$ calculates 1-coverage probability for a network with Rayleigh fading (exponentially distributed with unit mean) and log-normal shadowing.

4. We present a change of variables stimulated by the dimensional spherical coordinates.

$\mathrm{s} 1=\mathrm{u}[\sin \theta 1 \sin \theta 2 \ldots \sin \theta \mathrm{n}-1] 2 / \beta$

$s 2=u[\cos \theta 1 \sin \theta 2 \ldots \sin \theta n-1] 2 / \beta$

$\mathrm{s} 3=\mathrm{u} \cos \theta 2 \sin \theta 3 \ldots \sin \theta \mathrm{n}-1] 2 / \beta \ldots$

$\mathrm{sn}=\mathrm{u}[\cos \theta \mathrm{n}-1] 2 / \beta$.

Where $q i=q i(\theta i, \ldots, \theta n-1):=($ si $/ u) ~ \beta / 2$. When $\beta=2$ our scheme of synchronizes boils down to the regular $\mathrm{n}$ dimensional spherical coordinates, whose Jacobin is $\mathbf{J}^{-}(u, \theta 1$, $\ldots, \theta n-1)=u$ n-1 Qn-1 i=1 sini-1 $\theta \mathrm{i}$; $\operatorname{cf}[21$, eq. (1.5)]). By introduction (or element belongings and the chain rule) our coordinate system has the corresponding Jacobian Mathematical addition of hyper geometric function $2 \mathrm{~F} 1$ is used when the model has noise. A close-form answer with $2 \mathrm{~F} 1$ is used in the no noise case.

5. Simulation characters are also comprised for assessment resolves. All network base stations are experimented on a disk region. The disk region needs to be large sufficient to decrease "edge effects", which become more protruding when fading is included.

We assume noise power $-96 \mathrm{dBm}$ normalized by the base station power $62.2 \mathrm{dBm}$ which makes $\mathrm{W}=10-15.82$. We consider two values for the density of base stations: $\lambda=$ $4.619 \mathrm{~km}-2$, which corresponds to a "OLSR" network deployment and $\lambda=0.144 \mathrm{~km}-2$ for a "suburban" one. Figure 1 shows the distribution function of ECOSR from the strongest base station for both scenarios. We validate our approach by showing that the obtained results coincide with those of simulation with the latter approach being less numerically stable and much more time-consuming.

\section{RESULT}

In this segment we assume that the propagation-loss of each base station $\mathrm{x} \in \Phi$ is further modified by a random change nodes variable Fx and equal to $1(|\mathrm{x}|) /(\mathrm{SxFx})$

where given $\Phi,\{\mathrm{Fx}\} \mathrm{x} \in \Phi$ is a collection of independent and identically distributed random variables, independent of shadowing $\{S x\} x \in \Phi$. In our paper we fluctuate minimum energy threshold and minimum distance from high capacity grid , i.e., that the generic fading variable $\mathrm{F}$ is exponential, with $\mathrm{E}[\mathrm{Fx}]=1$. MATLAB software is used to simulate the efficient coverage results.

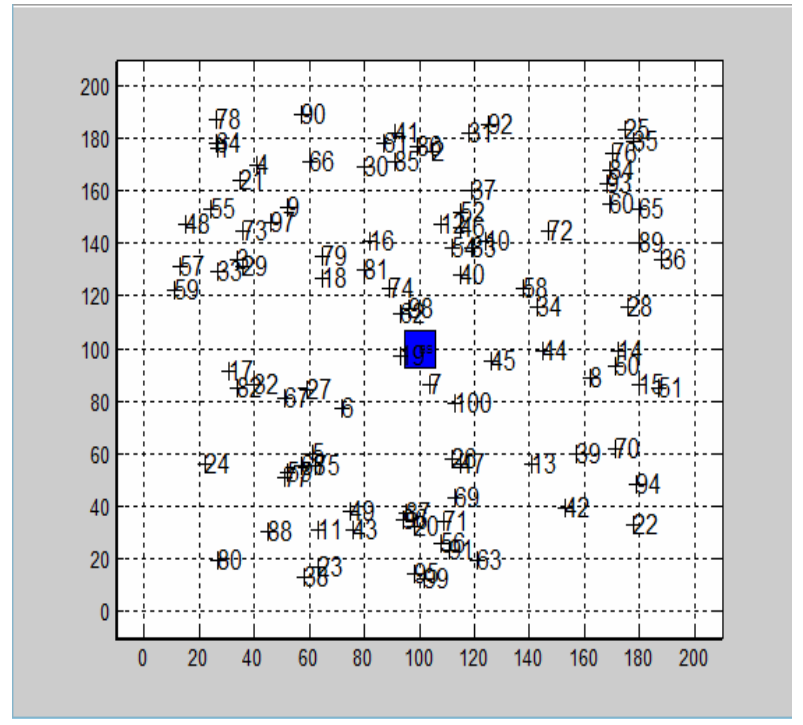

Figure 3: 100 Nodes with centric base station in Grid

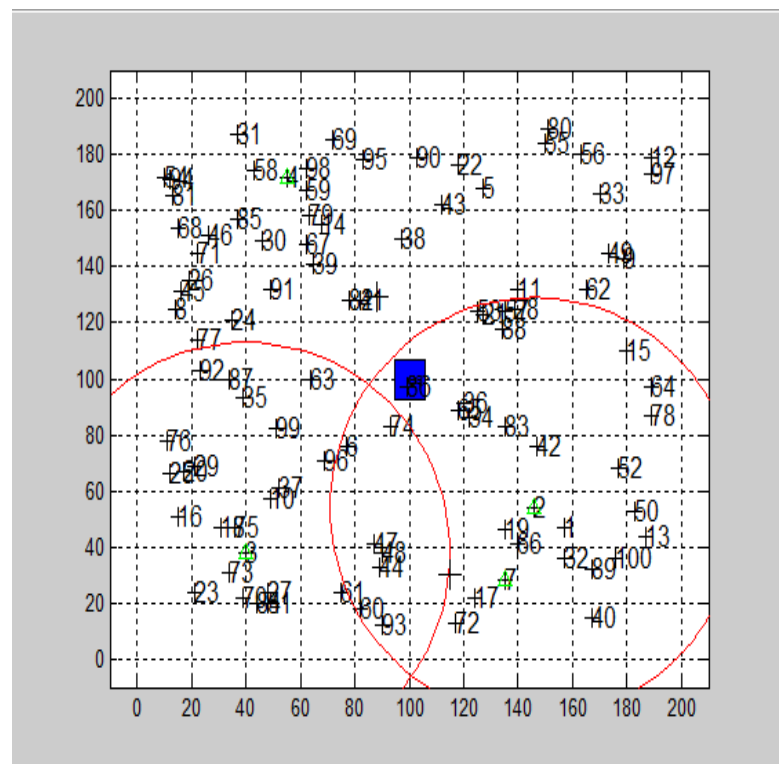

Figure 4: 100 Nodes with node shifting to void grid in 100 coverage range 


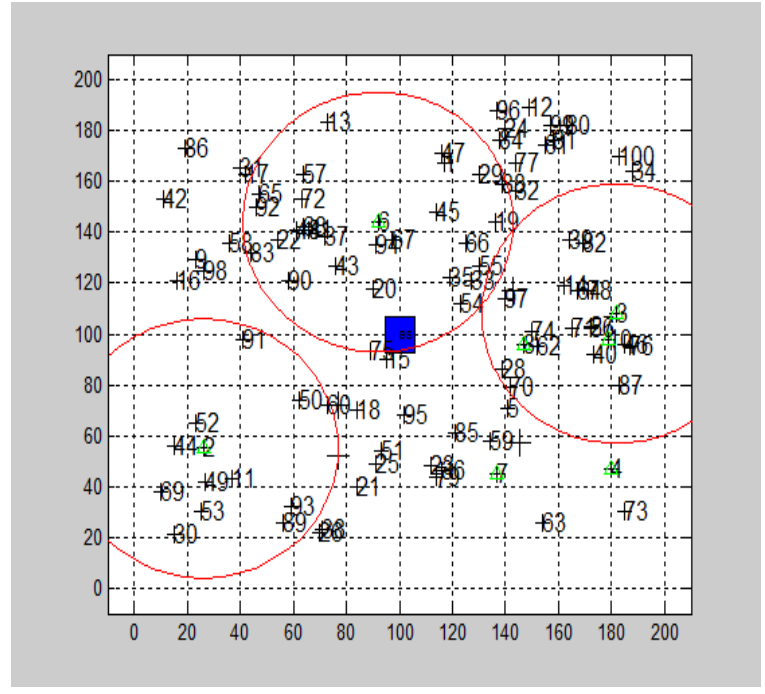

Figure 5: showing 3 lower energy node area in grid

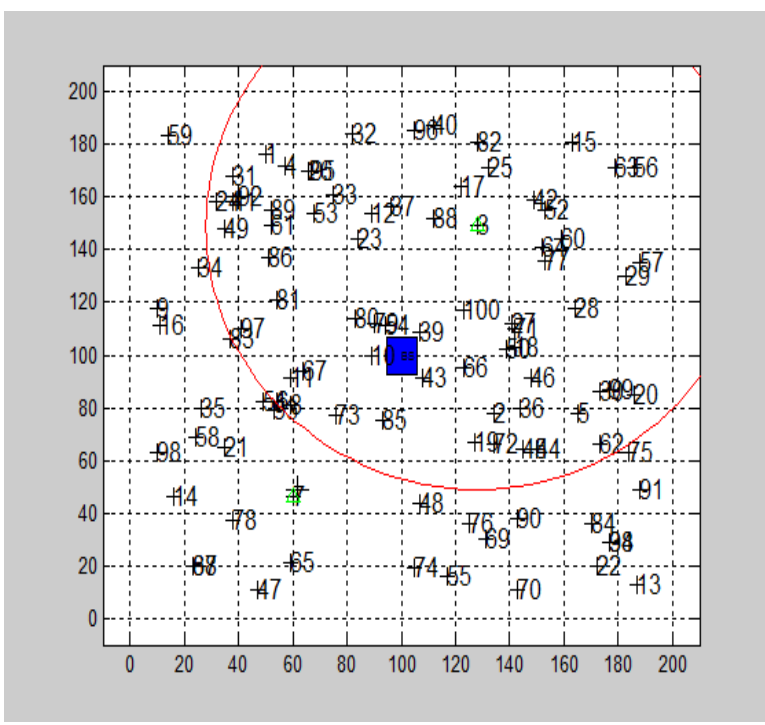

Figure 6: Covering to next void/ average coverage area which in minimum cover distance

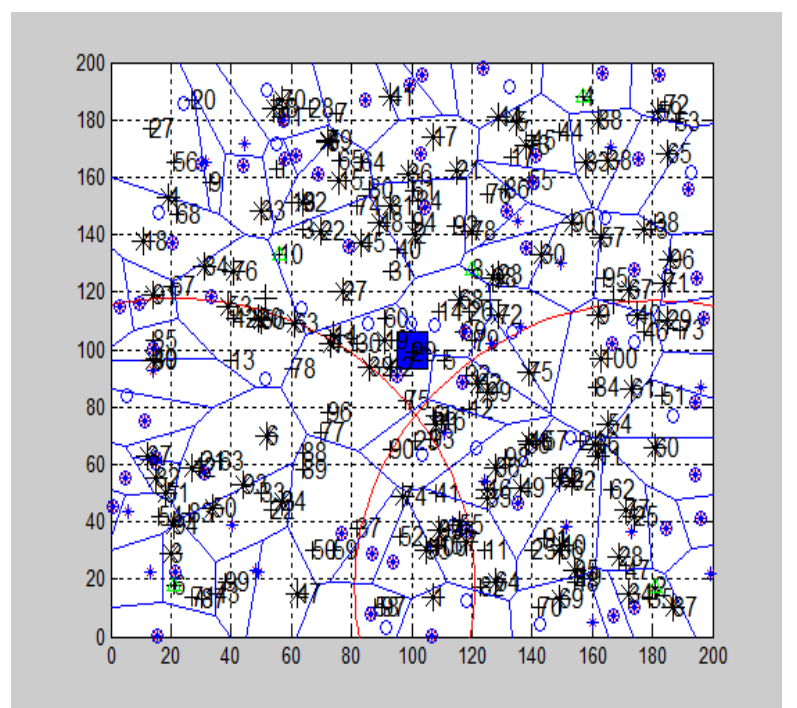

Figure 7: Execution coverage after shifting node by grid to grid

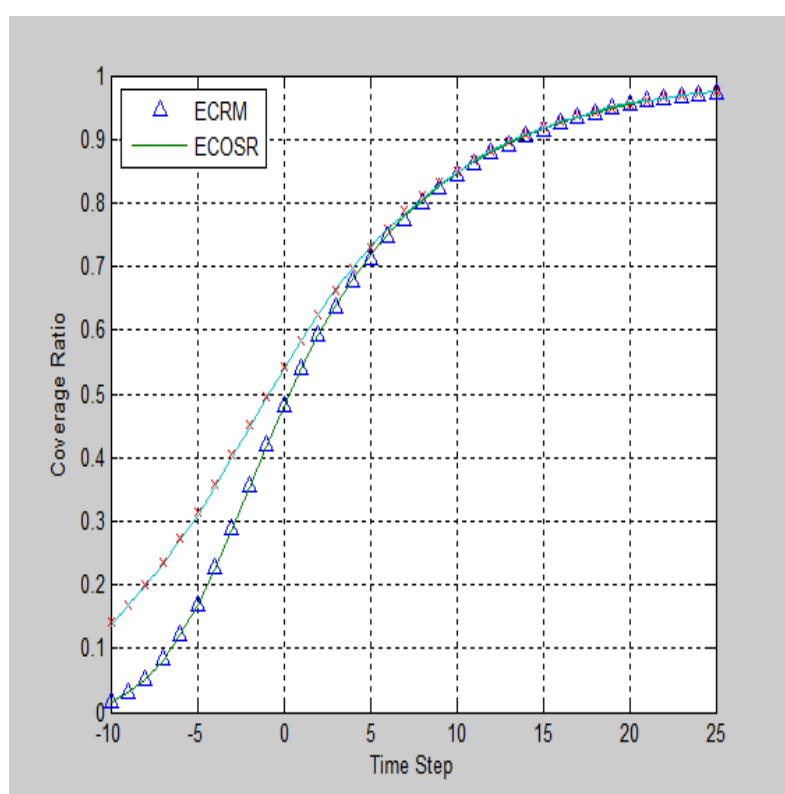

Figure 8: Showing Improvement Coverage ratio for ECOSR comparatively to Energy Coverage ratio

\section{CONCLUSION}

This paper enhanced routing protocol ECRM with proposed ECOSR (energy coverage overlap sensing ratio) for mobile WSNs where fixed nodes are merged with the mobile node in higher energy grid based node shifting and Control surroundings. The protocol arranges the fixed nodes into the communication backbone according to ECOSR(Energy coverage overlap sensing ratio) protocol we followed, which results in decrement of much energy consumption, distance based coverage, void area, overheads produced, and organizes the mobile nodes into Cluster which is the basic functioning cell of ECRM. ECRM chooses the maximum-rest-energy as the criterion to choose cluster head and makes mobile node as cluster head in order to delay the fixed nodes lifetime. The ECOSR protocol exceeds ECRM, SEP and LEACH at energy efficiency and robust of network lifetime and connecting higher coverage ratio in same network area at centric base station.

\section{REFERANCES}

[1] Raymond Mulligan et.al, April 2010. Coverage in wireless sensor networks: a survey network protocols and algorithms, vol. 2, no. 2, pp. 27-5.

[2] Chetan Chugh et.al, April 2013. A rea.l-time MATLAB based GUI for node placement and a shortest route path algorithm in wireless sensor networks", IJSETT ISSN No. (Online): 2250-3641, pp. 11-15.

[3] K. Martinez et.al, January 2004. GLACSWEB: a sensor web for glaciers, in proceedings of European workshop on wireless sensor networks, pp. 46-49, Berlin, Germany.

[4] James M. Gilbert et.al, October 2008. Comparison of energy harvesting system for wireless sensor network, International journal of automation and computing.

[5] W. M. Merrill et.al, March 2003. Collaborative networking requirements for unattended ground sensor systems, in proceedings of IEEE aerospace conference, IEEE press, vol. 5, pp. 2153-2165, Piscataway, USA. 
[6] Guang-Zhong Yang et.al , 2006. Body sensor networks, Springer-verlag, Germany.

[7] Brian Otis et.al, 2007.Ultra-low power wireless technologies for sensor networks, Springer.

[8] Bhuvaneswari P T V, 3 October, 2013. An energy efficient location based routing protocol for wireless sensor network, Anna University, Chennai.

[9] Weilian Su et.al, 2002. A Survey on sensor networks, IEEE communication magazine, vol. 40, pp. 102-116.

[10] Paolo Baronti et.al, "Wireless sensor networks: A survey on the state of the art and the 802.15.4 and ZigBee standards", Elsevier journal on computer communications, vol. 30, pp. 1655-1695, 2007.

[11] Nathalie Mitton et.al, "Balancing energy consumption in clustered wireless sensor networks", hindawi publishing corporation ISRN sensor networks, volume 2013, article ID 314732, 14 pages.

[12] Sajal K. Das et.al, "WCA: a weighted clustering algorithm for mobile ad hoc networks", cluster computing, vol. 5, 2002.

[13] Durga pavan nudurupati, Rajat kumar singh, 2013. Enhancing coverage ratio using mobility in heterogeneous wireless sensor network, Elsevier. 